\title{
Molecular Epidemiology of Chagas Disease in Venezuela
}

\author{
Carrasco $\mathrm{H} \mathrm{J}^{1 *}$, Nessi $\mathrm{AJ}^{1}$, Londono $\mathrm{JC}^{1}$, Rodriguez $\mathrm{AE}^{2}$, Moleiro $\mathrm{F}^{2}$ and Mendoza $\mathrm{I}^{2}$ \\ ${ }^{1}$ Laboratory of Molecular Biology of protozoa, Institute of Tropical Medicine, Faculty of Medicine, Central University of Venezuela, Venezuela \\ ${ }^{2}$ Section of Experimental Cardiology, Institute of Tropical Medicine, Faculty of Medicine, Central University of Venezuela
}

Received: November 29, 2013; Accepted: December 13, 2013; Published: December 21, 2013

*Corresponding author: Carrasco HJ, Laboratory of Molecular Biology of protozoa, Institute of Tropical Medicine, Faculty of Medicine, Central University of Venezuela, Venezuela, Tel: +58-212-6053546; Fax: +58-212-6053551; E-mail: hernan.carrasco@ucv.ve

\begin{abstract}
American Trypanosomiasis or Chagas' disease is endemic of the American continent with a wide distribution from the South of United States to North of Argentina. The etiological agent is the parasitic protozoan Trypanosoma cruzi which is transmitted by blood sucking triatomine bugs through contaminated feces. According to the last consensus meeting in 2009, T. cruzi has been divided in six groups or discrete typing units (DTU), also names genotypes, from TcI to TcVI. The geographical distribution of T. cruzi genotypes in the American Continent has some particularities. For instance, genotypes TcII, TcV and TcVI are found infecting humans at the Southern cone, whereas $\mathrm{TcI}$ and TcIV are the genotypes prevalent from the Amazon basin to Central and North America. In Latin America, for decades TIV was considered mainly sylvatic with very rare occasion of human infections. A recent study in Venezuela reported TcI, TcIII and TcIV circulating in domestic and sylvatic cycles, but only genotypes TcI and TcIV have been identified infecting humans in different regions of the country. Around $79 \%$ of the patients have been found infected with TcI and the remaining with TcIV. In this study we have made the follow up of 30 chronic chagasic patients showing that the clinical outcome of people infected with TcI shows to be more severe than those infected with TcIV, suggesting that TcI genotype is in general more pathogenic than TcIV. We have detected triatomine bugs and wild mammals naturally infected with TcIII, but no human infection with this genotype has been identified so far. Murine models indicate that T. cruzi isolates belonging to TcI genotype shows to be more virulent and pathogenic than TcIV. The biological behavior of TcI and TcIV isolates indicate a significant association with the clinical manifestation in the chronic patients.
\end{abstract}

Keywords: Trypanosoma cruzi; Genotypes; Chagas' disease; Chronic stage; Molecular typing

\section{Introduction}

Chagass disease or American trypanosomiasis, is caused by the hemoflagellate parasite Trypanosoma cruzi and represent in Latin America one of the main neglected tropical diseases. It was described for the first time in 1909 by the Brazilian physician Carlos Chagas. Considering that the disease mainly affects people living in poverty in rural or peri-urban areas of Latin America, it has not received the necessary attention. In 1981 the prevalence in Latin America was around 20 million people and has decreased in 2005 to 8-10 million, thanks to the vector control campaigns implemented in the Southern Cone, as well as the screening of blood donations in endemic areas. For several years the disease was considered circumscribed to the American continent, from the south of the USA to the north of Argentina and Chile, especially associated to poor rural areas. The migration of people from endemic regions of Latin America has been the cause of infection with Chagas' disease in non-endemic countries, mainly through blood transfusion, organ transplant and congenital transmission (from an infected mother to the newborn) [1]. This situation has drawn the attention of national health authorities in North America, Europe, Asia and Australia to establish more effective surveillance systems to prevent new cases of infections. The treatment available to date is poorly effective in the chronic phase of the disease and may produce in some cases, severe side effects due to toxicity of the drugs. In addition to lack of availability of the main drug for Chagas' disease, benznidazole, it has shown to be ineffective against the parasite in the chronic phase with important side effects in treated patients. At the moment, diagnosis, management and control of Chagas disease in endemic and non-endemic areas represent many challenges such as the absence of effective treatment for chronic cases, and there is no reliable marker to assess the cure after treatment. During its life cycle, the parasite can be transmitted to humans by infected triatomine bugs through the contaminated feces (vectorial transmission) and is one of the major parasitic diseases of Latin America. Other less frequent ways of transmission include transmission through consumption of food or drink contaminated with feces of infected triatomine bugs (oral transmission) and sharing needles by drug addicts [2]. Clinically, Chagas' disease can be divided into an acute phase and a chronic phase. The acute phase may involve symptoms as fever, headache, inflammation at the inoculation site (inoculation chagoma) or unilateral palpebral edema (Romana sign), splenomegaly and enlarged lymph nodes. Potential risk of mortality is associated with acute myocarditis and pericardial effusion. In most of the cases the acute phase resolves spontaneously in 2 to 4 months, after which the patient remains chronically infected. Majority of patients never develops symptoms and remain asymptomatic for decades. Some of them can develop symptoms characteristic of the chronic phase with cardiomyopathy, arrhythmias, megaesophagus and megacolon (megasyndromes). T. cruzi as specie shows a high genomic polymorphism which has been associated with wide variability 
in biological and biochemical characters. The first attempt of classification divided T. cruzi into two major groups, TcI and TcII; TcII was further divided into five subgroups (also called discrete typing units or genotypes) designated TcIIa to TcIIe [3]. In 2009, a consensus was reached so the six major recognized lineages will be renamed TcI to TcVI [4]. The TcI and TcII genotypes probably diverged from the same ancestral origin, whereas the $\mathrm{TcV}$ and $\mathrm{TcVI}$ seem to be emerged from at least two hybridization events [5]. The origins of TcIII and TcIV are still in discussion. One theory proposes TcIII and TcIV as ancestral strains [6]. A second theory consider both genotypes as the result of hybridization between TcI and TcII, which after a long evolution process becomes homozygous lineages sharing genetic characters with TcI and TcII [5]. The TcI genotype is present from South USA to North of Argentina and Chile in both domestic and sylvatic cycles. TcI seems to have evolved with arboreal opossums and vectors of the triatomine tribe Rhodniini [7]. Didelphis spp. are found infected with TcI all across the American Continent, however TcI has also been found infecting many others mammals $[8,9]$. $\mathrm{TcI}$ is the main genotype in Chagasic patients, from the Amazon basin to the south of North America [9-13]. TcIII is particularly distributed in South America and basically restricted to sylvatic cycles. The natural hosts for TcIII are armadillos, mainly Dasypus novemcinctus $[7,14]$. TcII, TcV and TcVI are the main genotypes found in human infections at the southern cone of the Continent [6], and are the genotypes associated with the domestic vector Triatoma infestans. Megasindromes and cardiomyopathy have been associated with $\mathrm{TcV}$ and $\mathrm{TcVI}$ in chagasic patients from Argentina, Chile, Bolivia, and Brazil. Megasindromes is practically absent upwards the Amazon basin, where predominates the genotype TcI $[15,16]$. In Bolivia and Argentina $\mathrm{TcV}$ is the most common genotype in newborn from mother with Chagas' disease. TcIV is found in South, Central and North America in several natural hosts. The sylvatic hosts include primates, arboreal mammals, rodents and terrestrial marsupials [7,17]. For decades very few cases of human infections with TcIV were identified, but recently an important prevalence of TcIV has been reported in chagasic patients in Venezuela [9]. In the present study we have identified the genotype of $95 \mathrm{~T}$. cruzi isolates obtained from the same number of chronic chagasic patients in Venezuela. All patients come from different regions of the country and the clinical evolution of 30 of them was analyzed with the aim to elucidate any possible relationship between the genotype of the infecting parasites and the progression of the disease. In same way, the biological behavior of two isolates representatives of the genotypes found in humans, were studied in mice with the intention of investigate if there was any correlation with the clinical condition observed in the infected patients.

\section{Materials and Methods}

\section{Patients population}

We chose 30 chronic chagasic patients that attended the cardiology unit at the Institute of Tropical Medicine and were positive with parasitological test through xenodiagnostic. Each patient had positive serology for Chagas' disease. With the aim to evaluate the clinical evolution of the patients, each of them were selected based on the availability of clinical history and attended regularly the cardiology unit, at least once a year.

\section{Parasites and DNA extraction}

T. cruzi isolates were obtained from each chagasic patient and just after the initial growth of the parasites in culture medium, an aliquot of each isolate was preserved in liquid nitrogen. The remaining cells were immediately used for DNA extraction, and selected isolates were additionally cloned as previously reported by Carrasco, et al. [9].

\section{Molecular typing of $\boldsymbol{T}$. cruzi isolates}

For the genotyping of T. cruzi isolates we used the PCRRFLP technique reported by Westenberger, et al, Rozas, et al., Carrasco, et al. $[5,9,18]$. The selected genes for PCR amplification were GPI (glucose phosphate isomerase), glycoprotein 72 (gp72) and the intergenic region of heat shock protein 60 (HSP60). The restriction enzymes used for the digestion of amplified products were Hha I, Taq I and EcoR V respectively. PCR and digestion products were separated by electrophoresis in $2.5 \%$ agarose gel and visualized with ethidium bromide staining under UV light, as reported by Carrasco et al. [9].

\section{Clinical conditions of chagasic patients}

In order to assess the Chagas' disease stage for each patient, we have considered the standard parameters to evaluate every case. Patients with cardiac pathologies not associated to Chagas' disease were not included in this study. According to the clinical information and following the classification reported by Garcia et al. [19], the stage of the chagasic patients were designated as Chagas I, II and III, as follows:

Chagas I (ChI): ELISA positive test (IgG), xenodiagnostic positive test, no evidences of cardiac damage (cardiopathy). Prognosis of life conditions of patient as normal population.

Chagas II (ChII): ELISA positive test (IgG), xenodiagnostic positive test, evidence of myocardial damage, in general no further symptoms presents.

Chagas III (ChIII): ELISA positive test (IgG), xenodiagnostic positive test, severe cardiac damage and congestive cardiac failure.

\section{Biological behavior of TcI and TcIV isolates in murine model}

One isolate representative of TcI genotype (X10462) and one representative of TcIV genotype (X11494) were used to infect NMRI. Two groups of mice were inoculated via intraperitoneal (ip) with trypomastigote forms of $T$. cruzi corresponding with one the genotypes under study. We also have included a control group inoculated i. p. with sterile saline solution. In order to study the virulence and pathogenicity of each genotype, we have evaluated the following parameters: pre-patent period, parasitemia, mortality, histopathological damage and tissue tropisms, following the methodology reported by Brener Z., Carneiro, et al. , Devera, et al. and Toledo, et al. [20-23]. 


\section{Results}

\section{Genotypes of $T$. cruzi isolates and clinical evolution of chagasic patients}

Thirty (30) T. cruzi isolates obtained from chagasic patients were analyzed with the PCR-RFLP technique, showing that 22 $(73.3 \%)$ patients were infected with TcI and $8(26,3 \%)$ with TcIV Table $1 \& 2$. The patients come from 15 States $(n=24)$, including the main endemic areas of the country, in a geographical extension that range from the western, central to the eastern parts of Venezuela. This means that we have included in our study patients with Chagas disease from regions all across the country (Table 1). With the aim to evaluate the development of Chagas' disease in each patient we have considered the initial and last information available in the clinical history of each patient at the Cardiology Unit. All patients were evaluated by electrocardiogram (EEC), echocardiogram (ECO), chest ray (RX) and Holter. As a result of the clinical study we found that of 14 patients initially classified as Chagas I, 1 of them evolved to Chagas II in 17 years.
In the same way, from 11 patients initially classified as Chagas II, 6 of them evolved to Chagas III in a period of 4 to 10 years (Table 1). It is important to notice the 5 patients were diagnosed as Chagas III since the initial clinical evaluation. When we look at the genotypes of the T. cruzi infecting the patients, we found that from 13 patients with the clinical condition of Chagas I, 6 of them were infected with TcI and 7 with TcIV $(n=13)$. In the case of patients with Chagas II, 5 were infected with TcI and 1 with TcIV $(\mathrm{N}=6)$. It is very important to highlight that 11 patients with Chagas III were infected only with TcI Table $1 \& 2$.

According to Table 1, no clear association was observed between the age and sex of the patients with the clinical condition of the Chagas' disease. As can be seen in Table 2, there is a clear relationship between the evolution of the clinical condition in chagasic patients and severity of the Chagas' disease with the genotype of the infecting parasites. As can be seen in Table 3, the chi square shows that the clinical condition of chagasic patients is associated with the T. cruzi genotype. In the same way, Odds ratio reveals that patients infected with TcI have higher risk of

Table 1: Clinical condition of chagasic patients and T. cruzi genotypes.

\begin{tabular}{|c|c|c|c|c|c|c|c|}
\hline Isolate & $\begin{array}{c}\text { Geographical origin } \\
\text { (State) }\end{array}$ & Age & Sex & Genotype & ICC & FCC & Period of Study \\
\hline X8120 & Tachira & 46 & $\mathrm{~F}$ & TcI & ChIII & ChIII & 1997-2001 \\
\hline X11881 & Anzoategui & 33 & M & TcI & ChIII & ChIII & 1990-2008 \\
\hline$X 11834$ & Monagas & 50 & M & $\mathrm{TcI}$ & ChIII & ChIII & 2003-2007 \\
\hline$X 10682$ & CB. Colombia* & 46 & $\mathrm{~F}$ & $\mathrm{TcI}$ & ChIII & ChIII & 2003-2005 \\
\hline$X 11541$ & Mérida & 53 & M & $\mathrm{TcI}$ & ChIII & ChIII & 2003-2005 \\
\hline$X 11250$ & Merida & 41 & M & TcI & ChII & ChIII & 1994-2003 \\
\hline X8396 & Portuguesa & 40 & $\mathrm{~F}$ & TcI & ChII & ChIII & 1999-2008 \\
\hline X8104 & Miranda & 54 & $\mathrm{~F}$ & TcI & ChII & ChIII & 2005-2009 \\
\hline X9354 & Sucre & 72 & M & $\mathrm{TcI}$ & ChII & ChIII & 1993-2001 \\
\hline$X 11713$ & Lara & 55 & M & TcI & ChII & ChIII & $2002-2008$ \\
\hline$X 11272$ & Portuguesa & 55 & M & TcIV & ChII & ChII & 1999-2004 \\
\hline X11704 & Anzoategui & 59 & $\mathrm{~F}$ & $\mathrm{TcI}$ & ChII & ChII & 2003-2008 \\
\hline$X 10827$ & Guárico & 61 & M & $\mathrm{TcI}$ & ChII & ChII & 2004-2007 \\
\hline$X 12914$ & Miranda & 55 & $\mathrm{~F}$ & $\mathrm{TcI}$ & ChII & hII & 2004-2010 \\
\hline$X 10868$ & Carabobo & 45 & $\mathrm{~F}$ & $\mathrm{TcI}$ & ChI & ChII & 1990-2007 \\
\hline$X 11302$ & Sucreá & 51 & M & TcI & ChI & ChI & 2003-2004 \\
\hline$X 12256$ & Trujillo & 51 & M & TcI & ChI & ChI & 1998-2006 \\
\hline$X 11006$ & Cojedes & 63 & $\mathrm{~F}$ & TcI & ChI & ChI & 2000 \\
\hline$X 11124$ & Dtto Federal & 59 & $\mathrm{~F}$ & TcI & ChI & ChI & 2003-2007 \\
\hline$X 11804$ & Portuguesa & 51 & M & TcI & ChI & ChI & 1991-2002 \\
\hline$X 10141$ & Trujillo & 61 & M & TcI & ChI & ChI & 1992-2007 \\
\hline$X 11494$ & Guárico & 48 & M & TcIV & ChI & ChI & $2002-2004$ \\
\hline X12087 & CB. Colombia* & 52 & $\mathrm{~F}$ & TcIV & ChI & ChI & 2002-2007 \\
\hline RM24 & Portuguesa & 30 & M & TcIV & ChI & ChI & 2004-2010 \\
\hline X8196 & Cojedes & 66 & $\mathrm{~F}$ & TcIV & ChI & ChI & 1998-2002 \\
\hline X9513 & Aragua & 60 & M & TcIV & ChI & ChI & 2001-2002 \\
\hline
\end{tabular}

ICC=Initial Clinical Condition, FCC=Final Clinical Condition.

${ }^{*} \mathrm{CB}$ Colombia $=$ Colombian border with Venezuela at Tachira State 
Tabla 2: Clinical Condition.

\begin{tabular}{|c|c|c|c|c|}
\hline Genotype & ChI & ChII & ChIII & Total \\
\hline TcI & $6(20 \%)$ & $5(16.66 \%)$ & $11(36.66 \%)$ & $22(73.3 \%)$ \\
\hline TcIV & $7(23.33 \%)$ & $1(3.33 \%)$ & $0(0 \%)$ & $8(26.7 \%)$ \\
\hline Total & $13(43.33 \%)$ & $6(20 \%)$ & $11(36.66 \%)$ & $\mathbf{3 0}(\mathbf{1 0 0} \%)$ \\
\hline
\end{tabular}

Table 3: Chi square $\left(X^{2}\right)$ and Odds ratio for T. cruzi genotypes and clinical condition of chagasic patients.

\begin{tabular}{|c|c|c|c|}
\hline Genotype & ${ }^{*}$ No symptomatic & ${ }^{* *}$ Symptomatic & Total \\
\hline TcI & 6 & 16 & 22 \\
\hline TcIV & 7 & 1 & 8 \\
\hline Total & 13 & 17 & 30 \\
\hline
\end{tabular}

Odds ratio $=18,6 ; X^{2}=9,21(\mathrm{p}=0,0099)$

${ }^{*}$ No symptomatic $=\mathrm{ChI} ;{ }^{* *}$ symptomatic $=\mathrm{ChII}$ or $\mathrm{ChIII}$

develop Chagas II and Chagas III when compared to patients no infected with to this genotype. According to Table 3, it suggest that $\mathrm{TcI}$ is the genotype associated with the more severe clinical condition $(\chi 2=9.71 ; p=0.0099)$ and the Odds ratio shows that there is 18.6 times more probability to develop severe stage of Chagas disease with TcI in relation with TcIV. This last genotype seems to be associated with the mild phase of the Chagas' disease (Chagas I).

\section{Infections of mice with TcI and TcIV genotypes}

With the purpose to compare the biological behavior of each genotype in murine models, we have inoculated i.p. three groups of NMRI mice. One group with the isolate X10462, representative of TcI genotype; a second group with the isolate X11494, representative of TcIV genotype; and a third group was inoculated with saline solution. Table 4 shows the results when NMRI mice were infected with either TcI or TcIV genotypes. It is quite clear that the isolate $\mathrm{X} 10462$, belonging to TcI genotype was highly virulent, with lethality of $100 \%$ in this mice strain. It is very important to notice that this isolate was obtained from a chagasic patient who was Chagas II in 1999 and in a period of 4 years evolved to Chagas III (Table 1). On the other site, the isolate X11494 showed very low infectivity and virulence. Even after 6 month of observation, this TcIV isolate did not kill any mice and scarce amastigote nests were detected in skeletal muscle (data not shown). The isolate X11494 was obtained from a chagasic patient with the clinical condition of Chagas I (Table 1). Another important result that reveals the pathogenic capacity of the TcI isolate, was the extensive damage generated in cardiac muscle of this mice, as shown in Figure 1a. In contrast, Figure 1b shows that TcIV isolate did not produced any apparent damage in the cardiac muscle.

\section{Discussion}

The geographical distribution of the six T. cruzi genotypes (TcI to TcVI) in the American Continent, have particular characteristics for some genotypes. For instance, TcII, TcV and TcVI are the main genotypes found in the Southern Cone of South America, implicated in human infection and apparently associated to megasyndromes and congenital transmission. TcIII seems to be restricted to sylvatic cycle and it appears to be distributed only in South America. TcI is widely distributed through the American Continent, circulating in domestic and sylvatic cycles. However, human cases of infections with TcI are predominant from the Amazon Basin to South of USA. Similarly, TcIV shows a wide distribution in the American Continent, but very few cases of human infections with this genotype had been reported and it was thought to be mainly associated to sylvatic cycle [24]. In the past, very limited studies have reported the identification of T. cruzi genotypes (former zymodemes or lineages) from human in Venezuela $[24,25]$. These studies were focused on few cases

Table 4: Biological behavior of TcI and TcIV genotypes in NMRI mice.

\begin{tabular}{|c|c|c|c|c|c|c|c|c|}
\hline Isolate & Genotype & Parasitema & $\begin{array}{l}\text { Pre-patent } \\
\text { Period } \\
\text { (days) }\end{array}$ & $\begin{array}{l}\text { Highest } \\
\text { Parasitemia (in } \\
\text { 5ul) }\end{array}$ & Virulence & Mortality & $\begin{array}{c}\text { Histop } \\
\text { I/0 }\end{array}$ & $\begin{array}{c}\text { atholy } \\
0 / P\end{array}$ \\
\hline $\mathrm{X} 10462$ & TcI & Patent & 5 & $26.5 \times 10^{3}$ & High & $100 \%$ & $5 / 5$ & $4 / 5$ \\
\hline X11494 & TcIV & Sub-patent & n.d. & n.d. & Low & $0 \%$ & $4 / 5$ & $1 / 5$ \\
\hline
\end{tabular}

I/O: Inflammation infiltrates per organ; O/P: organs infected with parasites; n.d. = not detected in peripheral blood samples by microscope examination.
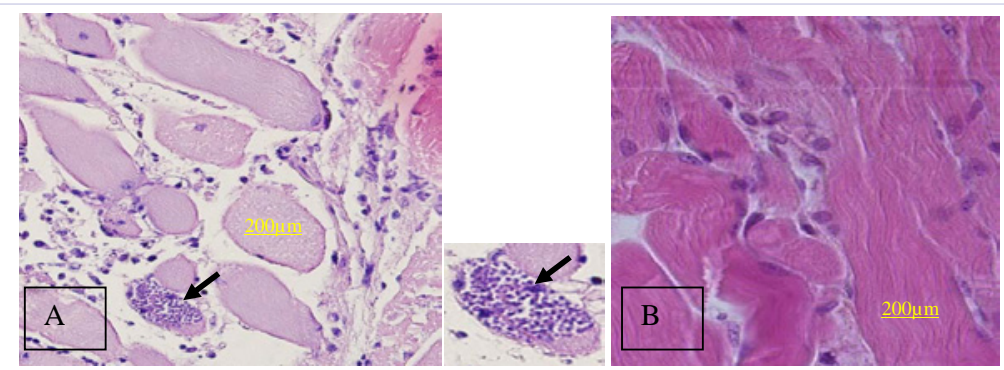

Figure 1: Cardiac muscle of NMRI mice infected with TcI and TcIV.

Cardiac muscle of NMRI mouse infected with:

A) X10462 isolate, showing amastigotes nest (arrow) and very intense inflammatory infiltrates with cardiac fiber destruction.

B) X11494 isolate, showing absence of amastigote nest and scarce inflammatory infiltrates. Magnification $=350 \mathrm{x}$. 
or a particular region of the country. Up to date, no relationship or association of genotypes with the clinical condition of chronic chagasic patients have been reported in Venezuela. A clear and well supported identification of $T$. cruzi genotypes circulating in Venezuela have been reported by Carrasco et al. [9]. The researcher have identified only TcI and TcIV in humans, beside there were able to identify TcIII genotype in natural reservoirs and sylvatic triatomine bugs. In the present study we have studied in a retrospective way, the clinical evolution of 30 chronic chagasic patients that attended the outpatient service of the Cardiology Unit at the Institute of Tropical Medicine, in Caracas, Venezuela. According to the clinical histories and medical examination of 30 patients, we found that 11 of them were Chagas III (33.66\%), and through molecular techniques we found that the infecting T. cruzi belong to the TcI genotype. 5 patients were diagnosed to be at the Chagas II stage (16.66\%) and being infected with TcI. One patient at the Chagas II stage (3.33\%) was infected with TcIV. This means that 16 symptomatic patients (Chagas II and Chagas III) were infected with TcI and only one symptomatic patient (Chagas II) was infected with TcIV. It is important to notice that none of the patients infected with TcI had no other co-infection. When we look at the no symptomatic patients, 6 were infected with TcI (20\%) and 7 with TcIV (23.33\%). Using this information, when we analyzed if there is any relationship between the genotype and the clinical stage of Chagas disease we found a clear association between the TcI genotype and symptomatic condition, with $\chi 2=$ $9.71(p=0.0099)$. The Odds ratio shows that there are 18.6 more probabilities to develop pathology in the chronic phase when the infection is caused by TcI genotype in comparison when the infection is caused by TcIV. As far as to our knowledge this is the first time that a representative number of cases of Chagas' diseases all across the country, where the clinical conditions of chronic chagasic patients have been associated with the genotype of infecting T. cruzi isolate in Venezuela. The biological behavior of two isolates representative of TcI and TcIV genotypes in NMRI mice, showed that TcI genotype was more virulent and pathogenic when compared with the TcIV genotype. One relevant characteristic of both isolates was the fact that TcI isolate was obtained from a chronic chagasic patient that presented the more severe condition of the disease (Chagas III), while the TcIV isolate was obtained from a chagasic patient at the no symptomatic stage of the disease (Chagas I). We found similar behaviors of others isolates belonging to TcI and TcIV isolates in murine model that could be associated with the clinical conditions of the chagasic patients (data not shown; Nessi, et al., manuscript in preparation), reinforcing the results shown in the present study. With the aim to analyze the population composition of the TcI isolate used in the present study, It was cloned using the technique reported by Yeo, et al. [26], and each clone subpopulation was characterized by the RAPD technique, showing that TcI isolate was very homogenous in their population compositions (data not shown), so it can be rule out that the behavior of the TcI isolate is due to different subpopulation of T. cruzi. Finally, in this study we have presented clear evidence that demonstrated that TcI genotype is associated with the more severe clinical condition and prognosis of Chagas' disease in Venezuela. These results might have important medical and epidemiological implications, in particular with the surveillance, prevention, diagnosis and control campaigns of Chagas' disease in Venezuela.

\section{Acknowledgements}

This study was conducted with the financial support of Grants FONACIT G2005000827 and PEI 2012001250.

\section{References}

1. Carlier, Y., Torrico, F., Sosa-Estani, S., Russomando, G., Luquetti, A., Freilij, H., et al (2011). Congenital Chagas disease: recommendations for diagnosis, treatment and control of newborns, siblings and pregnant women. PLoS Negl Trop Dis, 5(10), e1250.

2. Rassi, A., \& Marcondes de Rezende, J. (2012). American Trypanosomiasis (Chagas Disease). Infect Dis Clin N Am, 26(2), 275291.

3. Anonymous. (1999). Recommendations from a Satellite Meeting. Mem Inst Oswaldo Cruz, 94, 429-432.

4. Zingales, B., Andrade, S.G., Briones, M.R., Campbell, D.A., Chiari, E., Fernandes, O., et al (2009). A new consensus for Trypanosoma cruzi intraspecific nomenclature: second revision meeting recommends $\mathrm{TcI}$ to TcVI. Second Satellite Meeting. Mem Inst Oswaldo Cruz 104, 1051-4.

5. Westenberger, S.J., Barnabe, C., Campbell, D.A., \& Sturm, N.R. (2005). Two hybridization events define the population structure of Trypanosoma cruzi. Genetics, 171(2), 527-543.

6. Zingales, B., Miles, M. A., Campbell, D. A., Tibayrenc, M., Macedo, A. M., Teixeira M. M., et al. (2012). The revised Trypanosoma cruzi subspecific nomenclature: rationale, epidemiological relevance and research applications. Infect Genet Evol, 12(2), 240-253.

7. Yeo, M., Acosta, N., Llewellyn, M., Sanchez, H., Adamson, S., Miles, G. A., et al. (2005). Origins of Chagas disease: Didelphis species are natural hosts of Trypanosoma cruzi I and armadillos hosts of Trypanosoma cruzi II, including hybrids. Int J Parasitol, 35(2), 225-233.

8. Llewellyn, M.S., Miles, M.A., Carrasco, H.J., Lewis, M.D., Yeo, M., Vargas, j., et al. (2009). Genome-scale multilocus microsatellite typing of Trypanosoma cruzi discrete typing unit I reveals phylogeographic structure and specific genotypes linked to human infection. PLOS Pathogens, 5(5), e1000410.

9. Carrasco, H.J., Segovia, M., Llewellyn, M.S., Morocoima, A., UrdanetaMorales, S., Martinez, C.E., et al (2012) Geographical distribution of Trypanosoma cruzi genotypes in Venezuela. PLOS Negl Trop Dis, 6(6), e1707.

10. Bosseno, M.F, Barnabe, C., Magallon-Gastelum, E., Lozano-Kasten, F., Ramsey, J., Bertha, E. et al (2002) Predominance of Trypanosoma cruzi lineage I in Mexico. journal of Clinical Microbiology, 40, 627-632.

11. Iwagami, M., Higo, H., Miura, S., Yanagi, T., Tada, I., Kano, S., et al. (2007) Molecular phylogeny of Trypanosoma cruzi from Central America (Guatemala) and a comparison with South American strains. Parasitol Res, 102(1), 129-134.

12. Roellig, D.M, Brown, E.L., Barnabe, C., Tibayrenc, M., Steurer, F.J., \& Yabsley, M.J. (2008). Molecular typing of Trypanosoma cruzi isolates, United States. Emerging Infectious Diseases, 14(7), 1123-1125.

13.Samudio, F., Ortega-Barria, E., Saldana, A., \& Calzada, J. (2007). Predominance of Trypanosoma cruzi I among Panamanian sylvatic isolates. Acta Trop, 101(2), 178-181. 
14. Morocoima, A., Carrasco, H. J., Boadas, J., Chique, J. D., Herrera, L., \& Urdaneta-Morales, S. (2012). Trypanosoma cruzi III from armadillos (Dasypus novemcinctus novemcinctus) from Northeastern Venezuela and its biological behavior in murine model. Risk of emergency of Chagas' disease. Experimental Parasitology 132(3), 341-347.

15. Ruiz-Sanchez, R., Leon, M. P., Matta, V., Reyes, P. A, Lopez, R., Jay, D., et al. (2005). Trypanosoma cruzi isolates From Mexican and Guatemalan acute and chronic chagasic cardiopathy patients belong to Trypanosoma cruzi I. Mem Inst Oswaldo Cruz, 100(3), 67-72.

16. Espinoza, B., Rico, T., Sosa, S., Oaxaca, E., Vizcaino-Castillo, A., Caballero, M.L., et al.. (2010). Mexican Trypanosoma cruzi T.cruzi I strains with different degrees of virulence induce diverse humoral and cellular inmune responses in a murine experimental infection model.J Biomed Biotech.

17. Bern, C., Kjos, S., Yabsley, M., Montgomery, S. (2011). Trypanosoma cruzi and Chagas' disease in the United States. Clinical Microbiology Reviews, 24(4), 655-681.

18. Rozas, M., Botto-Mahan, C., Coronado, X., Ortiz, S., Cattan, P.E., \& Solari, A. (2007). Coexistence of Trypanosoma cruzi genotypes in wild and periodomestic mammals in chile. Am J Trop Med Hyg 77(4), 647-653.

19. Garcia, R., Hernandez, E., Rodriguez, C., Jimenez, M., Bonfante, R., Anez, N., et al. (2001). Primer consenso venezolano sobre la enfermedad de Chagas: Conclusiones y recomendaciones. Avanc Cardiol, 21, 14-23.

20. Brener, Z. (1962). Therapeutic activity and criterion of cure on mice experimental infected with Trypanosoma cruzi. Rev Inst Med Trop Sao Paulo, 4, 389-396.
21. Carneiro, M., Romanha, A., Chiari, E. 1991. Biological characterization of Trypanosoma cruzi strains from different zymodemes and schizodemes. Mem Inst Oswaldo Cruz, 86(4), 387-397.

22. Devera, R., Illamendi, X., Montoya-Araujo, R., Pirmez, C., Fernandes, O., \& Coura, J. R. (2002) Biodemas de cepas de Trypanosoma cruzi isoladas de humanos de tres areas endemicas de Minas Gerais. Rev Soc Bras Med Trop 35(4), 323-330.

23.Toledo, M., De Lana, M., Carneiro, M., Bahia, M., Machado-Coelho, G.L., Veloso, V.M., et al. (2002). Impact of Trypanosoma cruzi clonal evolution on its biological properties in mice. Exp Parasitol, 100(3), 161-172.

24. Miles, M. A., Cedillos, R. A., Povoa, M. M., de Souza, A. A., Prata, A., Macedo, V. (1981). Do radically dissimilar Trypanosoma cruzi strains (zymodemes) cause Venezuelan and Brazilian forms of Chagas' disease? Lancet, 1(8234), 1338-40.

25. Anez, N., Crisante, G., Anez-Rojas, N., Rojas, A., Moreno, G., da Silva, F. M., \& Teixeira, M.M.G. (2009). Genetic typing of Trypanosoma cruzi isolates from Different hosts and geographical areas of western Venezuela. Bol. Mal. Salud Amb. 49(2), 251- 258.

26. Yeo, M., Lewis, M. D., Carrasco, H. J., Acosta, N., Llewellyn, M., da Silva Valente, S. A., de Costa Valente, V., de Arias, A.R., Miles, M. A. (2007). Resolution of multiclonal infections of Trypanosoma cruzi from naturally infected triatomine bugs and from experimentally infected mice by direct plating on a ensitive solid medium. Int J Parasitol, 37(1), 111-120. 